\title{
Six-Phase VSI Control Using 8-Bit MCU
}

\author{
A. Pitrėnas, A. Petrovas
}

\begin{abstract}
In this paper a generation of six-phase voltage using six leg synchronous Voltage Source Inverter and a low cost 8-bit Micro-Controlling Unit is investigated. A simple and cost effective Sinusoidal Pulse Width Modulation for controlling sixleg VSI is presented. A simulation on generation of six-phase voltage using SPWM method was investigated. The main advantage of presented SPWM method is the simplicity and low resource requirement for $\mathrm{MCU}$.
\end{abstract}

Index Terms - Scalar control, Six-phase VSI, SPWM method.

\section{INTRODUCTION}

$\mathrm{T}$ HE interest of multi-phase drives has grown over the years. The main reasons for that are their advantages over regular drives. Multi-phase drives have less torque ripple, greater reliability, fault tolerant operation and some other advantages compared with three-phase drives. Multi-phase drives require multi-phase Voltage Source Inverters to form a multi-phase voltage for their supply. Multi-phase drives are widely used in electric cars, ships, locomotives and aircrafts. [1] Multi-phase VSI's have different count of so called legs depending on phase count, thus the control of multi-phase VSI's are different.

There are two main methods of induction motor control. First is so called scalar control (in some literature it's called $\mathrm{V} / \mathrm{Hz}$ control). When scalar control is used the magnitude and frequency of motor's voltages are controlled. The implementation of this method is simple, but the torque response of this method is poorer compared to other methods.

Other control method is called vector control (or Field Oriental Control). When FOC is used it controls phase of motor's current, its amplitude and frequency. This method provides fast torque response. The Direct Torque Control is derived from FOC method. [2] - [3] Vector control is utilized using Space Vector Pulse Width Modulation technique.

The full SVPWM technique is somewhat more complex compared Sinusoidal PWM used in scalar methods and it requires more than ordinary MCU. Mainly the use of Digital Signal Processors or even Field Programmable Gate Arrays or an Application-Specific Integrated Circuits are required. [4] And this raises the complexity and price of the final project or device.

A. Pitrenas is with the Department of Automation of Vilnius Gediminas technical university, Vilnius, 03227 Lithuania (e-mail: aurelijus.pitrenas@vgtu.lt).

A. Petrovas is with the Department of Automation of Vilnius Gediminas technical university, Vilnius, 03227 Lithuania (e-mail: andrius.petrovas@vgtu.1t).
A lot of methods regarding simplification of the complex FOC of multi-phase drives can be found. This is mainly done to lower required MCU or DSP resources. Some methods go so far that the needed MCU resources for multi-phase SVPWM control are equal to resources required for threephase SVPWM. Hoverer the majority of these methods balance between needed resources of MCU and the purity of VSI output voltages. [5] - [6]

Summarizing both control methods have their advantages and disadvantages. FOC method controls motors currents so its operate with fast responses. This method is used when motor runs in transient operation; it's a high-performance control technique. Its disadvantage is high price and complexity of driver circuit.

On the other hand scalar control is cheap and well implementable method. It has some disadvantages when dealing with drives that operate with dynamic behaviour, since it gives low responses to transients. Its performance is low, but the technique itself is very stable. Both techniques are applicable over the nominal speed at the expense of torque. [7] This paper will focus on generation of SPWM signals for sixphase VSI control using a low cost 8-bit MCU. Later developed method could be used to develop a scalar control of six-phase induction motor.

\section{Generation Of SiX-Phase Voltage}

To form a six-phase voltage a six-leg Voltage Source Inverters are used (Fig. 1). One leg of VSI is formed using two solid state switches (most often Insulated Gate Bipolar Transistors). These legs are connected to DC bus. DC bus voltage is supplied from AC line using full bridge rectifiers and decoupling capacitors. Load is connected between legs middle points. When VSI is controlled in synchronous mode, the switches of one VSI leg turns on and off synchronously ex. when S1 is on, S7 is turned off. When every leg of VSI is switched in right order the six-phase voltage is fed to the load.

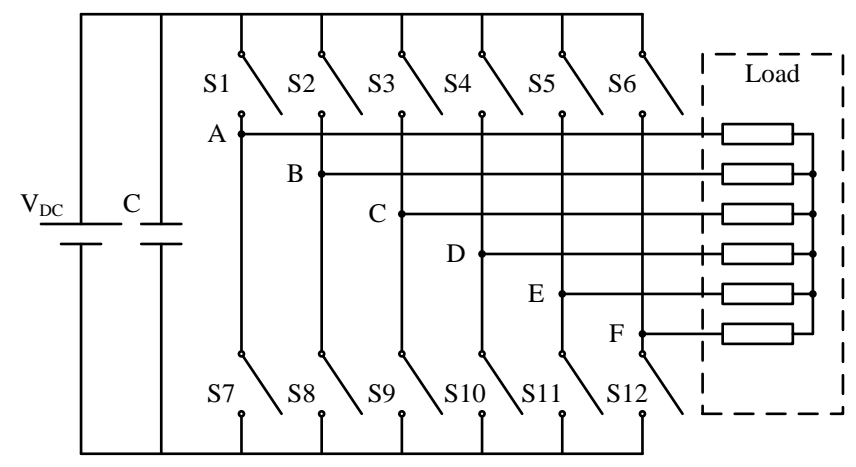

Fig. 1. Two level six-phase VSI 
The use of synchronous VSI leads to lower requirements of the MCU and this brings the cost of the system down. For controlling a synchronous six-phase VSI a total of six Pulse Width Modulation outputs of MCU are required. PWM outputs must be formed in the way that they correspond to signal which varies according to sinus function. This method is called Sinusoidal PWM (SPWM) or carrier based PWM. This method is illustrated in Fig. 2.
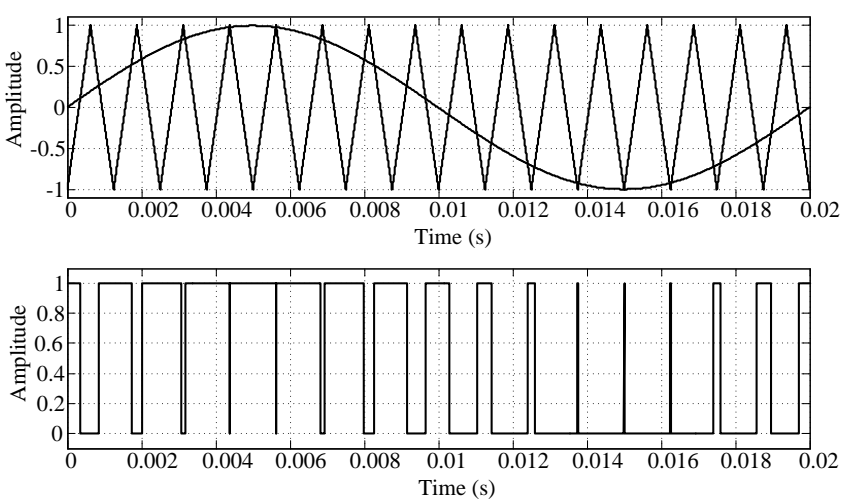

Fig. 2. Generation of carrier based SPWM signal

Carrier based PWM formation works using simple principles: A sinusoidal varied signal is compared to a higher frequency triangle signal, while amplitude of sinusoidal signal is higher than amplitude of triangle signal the output of PWM port is set to HIGH. When amplitude of sinusoidal signal is lower than amplitude of triangle signal, PWM port is set to LOW. The frequency of triangle signal is called carrier frequency. It's should be mentioned that the higher the carrier frequency the less distortions are brought to sinusoidal output. If the amplitude of final VSI output has to be changed then the amplitude of sinusoidal signal is changed while amplitude of triangle signal is kept constant. In the past this type of control algorithm was carried out using analog circuits and in our days this is accomplished using MCU's and DSP's.

\section{SIX-PHASE SPWM IMPLEMENTATION WITH 8-BIT MCU}

A six-phase voltage is shown in Fig 3. To form a six-phase voltage with VSI leads to calculation of six sinus functions by the MCU, but the low cost MCU are incapable of this task, thus the more powerful MCU or DSP should be used, or some simplifications should be carried out.

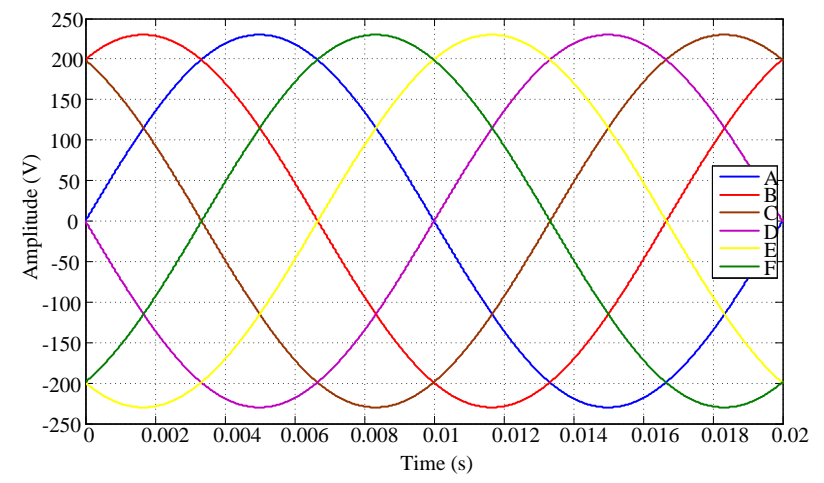

Fig. 3. Six-phase voltage
First simplification comes from that, that all six phases are the same only with $60^{\circ}$ degree angle shift between them. This means that MCU has to generate only one SPWM signal and all other can be generated by shifting the first one. Even the low coat modern MCU's have built-in hardware PWM generators which dramatically eases the generation of VSI control signals. Basically the required pulse width of PWM signal is set by writing needed value to according PWM control register.

By using a synchronous VSI control signals of one VSI leg is formed using one PWM channel. The PWM register value has to be changed in every new angle of generating SPWM function. To calculate the needed PWM register value depending on sinus angle following equation is used:

$$
R_{v}=\sin (\alpha) \cdot R_{R}
$$

where: $R_{v}$ - register value; $\alpha$ - phase angle in radians; $R_{R}-$ resolution of register (if register's resolution are 8-bits, then $R_{R}=255$ ).

However the calculated value $R_{v}$ will be floating number and operations with floating numbers in 8-bit MCU's take longer times and moreover the floating numbers can't be used in 8 bit register. These limitations leads to the need of rounding value $R_{v}$, that it could be used as integer type variable. One more thing should be mentioned that when angle of sinus signal goes over $180^{\circ}$ degree, the calculated PWM register's value will be negative and this is not acceptable.

By looking at the one phase sinus signal (Fig. 4) some more simplifications could be done. First of all we notice that sinusoidal signal is symmetrical. This means that we don't need to calculate angles between $180^{\circ}$ and $360^{\circ}$ all we have to do is to invert calculated values for angles from $0^{\circ}$ to $180^{\circ}$. The simplification of sinus calculation is already a meaningful but this could go a little bit more. In the same manner as before we can see that sinusoidal signal is symmetrical between $0^{\circ}$ to $90^{\circ}$ degrees and $90^{\circ}$ to $180^{\circ}$ degrees. This leads to fact that $\mathrm{MCU}$ has to calculate sinus only for angles from $0^{\circ}$ to $90^{\circ}$ and all other needed values could be found by inverting an already calculated ones.

The calculation of all needed PWM registers values of sinus angles could be done in two different approaches. First one is to calculate all 91 values (angle is varied from 0 to 90 including 0) sinus when MCU is turned on, every time, and then store them in Random Access Memory. But this step wastes a big amount of RAM which is critical in low cost MCUs. Second approach is to calculate all needed values in Personal Computer and then store them in MCUs program memory together with the main program. This approach is a lot better because we don't utilize a lot of RAM for variable storage and the MCU don't need to calculate sinus functions at all.

For calculation of all needed values of PWM registers this Matlab script could be used:

angle_deg $=(0: 90)$;

angle_rad $=$ anlge_deg*(pi/180); 
sin_rad $=\sin ($ angle_rad $)$;

sin_pwm = sin_rad $* 255$;

spwm $=\operatorname{round}\left(\sin \_p w m\right)$;

Array spwm is then transferred to program memory of MCU together with the main program. The main program of MCU is mainly interrupt based. The values for all six PWM register are transferred using one function. This function is called from timer interrupt routine. Depending on register value of this timer the interrupt is called in different times and this leads to a change of generated six-phase voltage's frequency.

The amplitude is changed by dividing PWM register's value according to the generating frequency of six-phase voltage.

To take this approach one step further one more simplification can be achieved easily, which leads to lowering the needed PWM output of MCU by factor of 2 . If we one more time thoroughly look at six- voltage diagram, we can see that some phases are shifted by angle of $180^{\circ}$ degree. This leads to the fact, that three voltages are shifted by angles of 60 ${ }^{\circ}$ degree and other three is inversion of first ones. This means that MCU could generate only three SPWM signals with phase difference of $60^{\circ}$ degrees, and other phases could be obtained by inverting them. This could be carried out in a hardware level of VSI. For example VSI's first leg's upper switch control signal could be connected to fourths leg's lower switch and first leg's lower switch signal should be connected to fourths leg's upper switch. If we do that for all other legs of VSI, the total required SPWM control signal count drops to 3 . This means that six-phase voltage can be generated using the really low end MCU and this is exactly what this paper focuses on. The algorithm of this SPWM generation method is shown in Fig. 4.

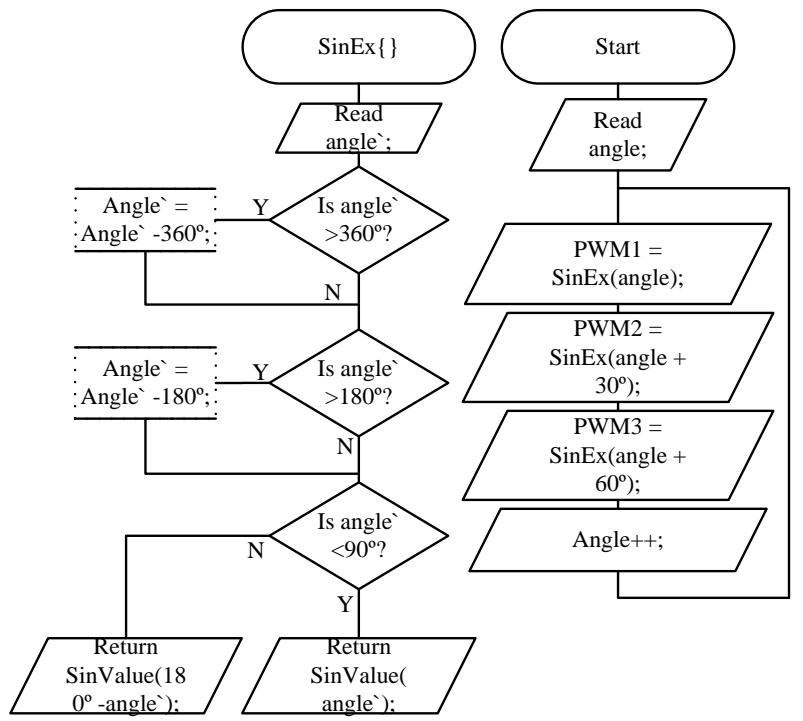

Fig. 4. Algorithm of SPWM signal generation using 3 PWM channels

\section{MODELLING}

In this paper modelling of SPWM six-phase voltage formation is carried out using Matlab Simulink (Fig. 5). The simulation model consists of three main blocks: generation of SPWM signals, six-phase VSI and 2nd order low pass filter.
SPWM signals are generated using carrier base PWM method, because PC have enough resources to calculate sinus functions quick enough and there is no need to simplify the generation of control signals as in case of MCU's. Six-phase VSI is modelled using ideal switches connected to DC bus voltage. 2nd order low pass filter is used to filter the output waveforms of VSI, this block can be found in SimPowerSystems toolbox. The filter's cut-off frequency is set to $200 \mathrm{~Hz}$.

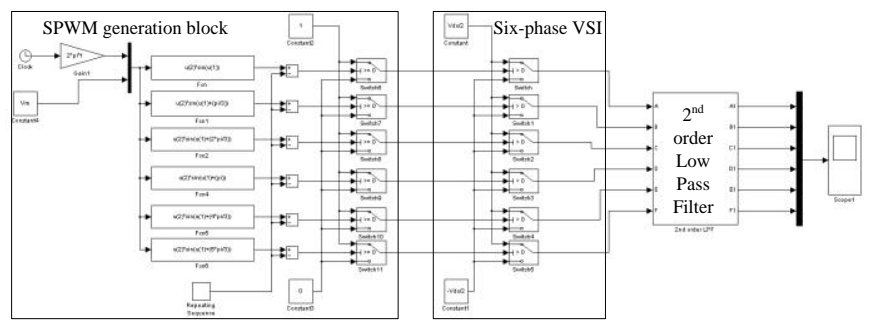

Fig. 5. Matlab Simulink model of idealized six-phase VSI with SPWM control

In Fig. 6 a $50 \mathrm{~Hz}$, six phase line to neutral voltage is shown, when voltage of DC bus is set to $200 \mathrm{~V}$ and carrier frequency to $2 \mathrm{kHz}$.

The control SPWM signals for two VSI legs are shown in Fig. 7.

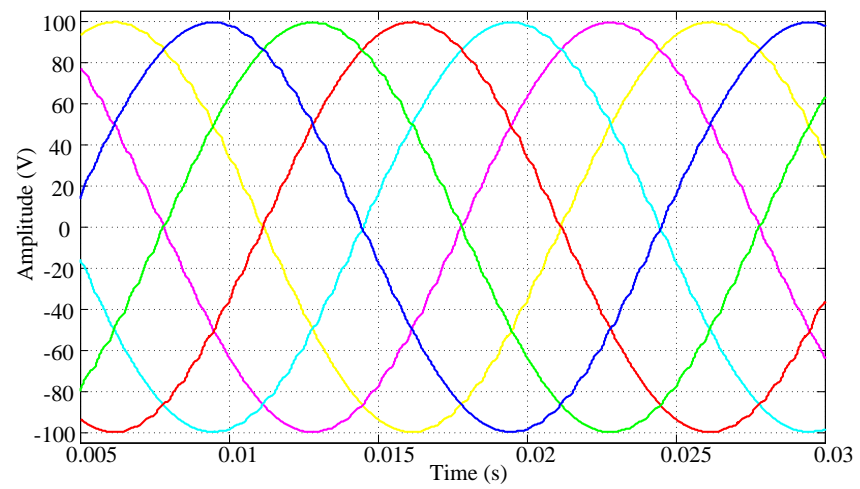

Fig. 6. Modelled six-phase voltage using SPWM method when DC bus voltage is set to $200 \mathrm{~V}$.
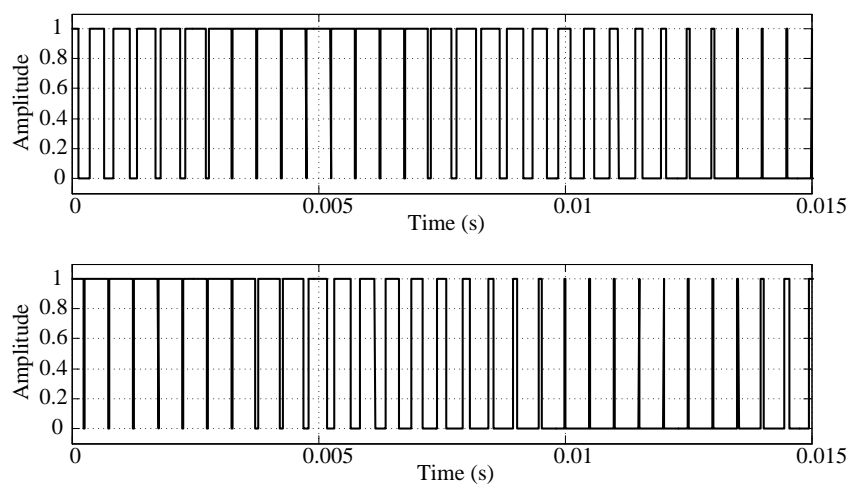

Fig. 7. Modelled SPWM signals for controlling two legs of six-phase VSI

By analysis of modelling results it is clear that simplest SPWM control method of VSI can be used to generate sixphase voltage and moreover the output voltages are almost pure sinusoidal with some switching harmonics which cannot be avoided by any control algorithm which involves any type of switching of VSI legs. 


\section{EXPERIMENTAL RESULTS}

The method of generating SPWM control signals for sixphase VSI described in section III was implemented using Atmel's 8-bit MCU Atmega328. A two SPWM control signals of six-phase VSI is shown in Fig. 8.

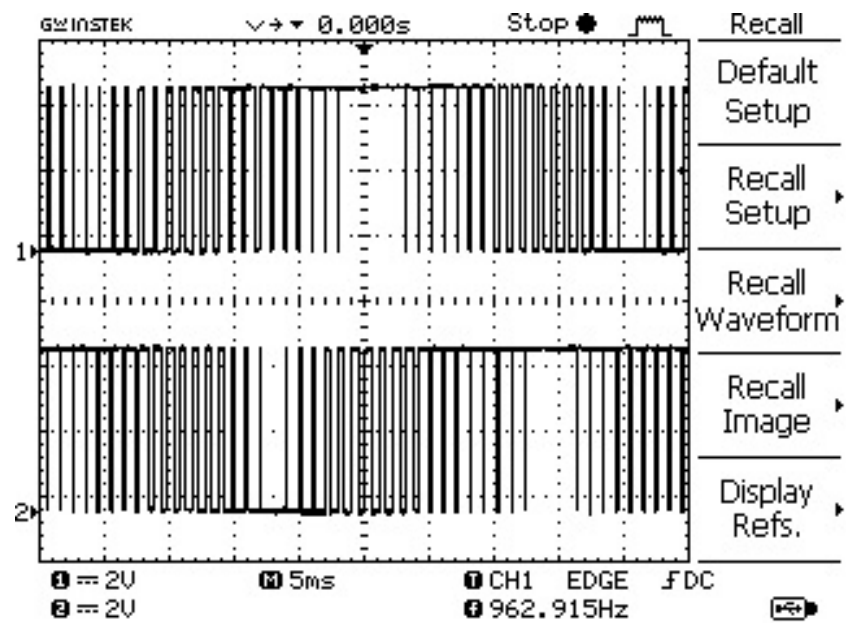

Fig. 8. Two SPWM signals generated with 8 -bit MCU shifted by $60^{\circ}$ degrees

These SPWM signals form a near $25 \mathrm{~Hz}$ output voltage are different by $60^{\circ}$ degree angle. Using such signals with accordingly connected VSI six-phase voltage can be generated. Two nearest line-to-line voltages would look as shown in Fig. 9.

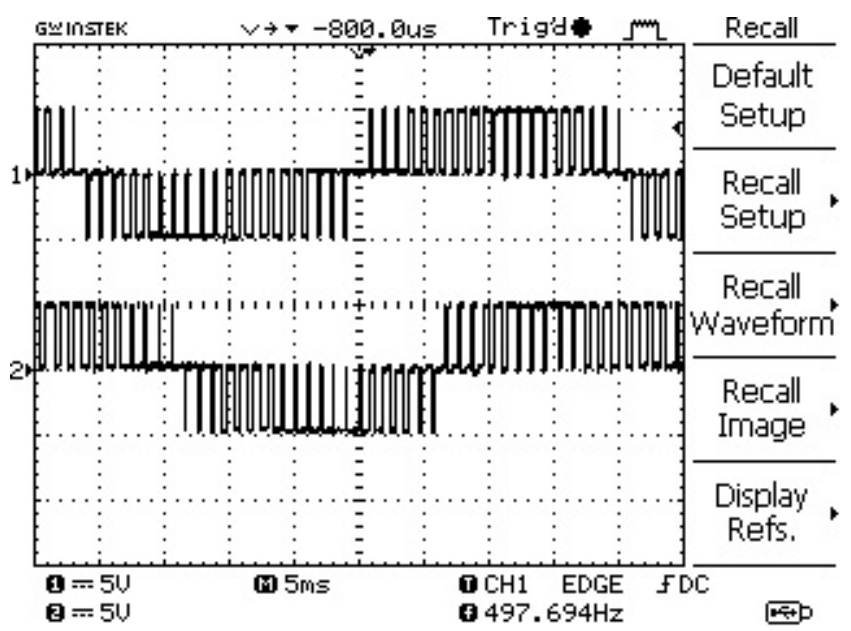

Fig. 9. Line-to-line voltages of VSI

\section{CONCLUSIONS}

In this paper a simple six-phase voltage generation method using simple and cheap 8-bit MCU are discuses and presented. As observed from modelling results a simple SPWM scheme could be used to form a nearly perfect sinusoidal six-phase voltage. This is important knowing the fact that, when sixphase voltage are generated using more sophisticated methods like SVPWM, the output waveforms may become more disturber when some simplification of method is implemented. It should be noted that these simplifications are needed for lowering the needed computations of MCU. If the simplifications are not used then the output voltage is near sinusoidal, but the complexity of control algorithm rises the need of powerful MCU, DSP or even FPGA and this rises the final cost of a project or a final device it's self.

In conclusion the proposed method will be studied more deeply to investigate the potential of the use of this method for a six-phase induction motor inverter. That type of inverter could be used in areas where torque control is not critical or not necessary at all and the cost of the inverter plays some important role.

\section{REFERENCES}

[1] G. Renukadevi, K. Rajambal. Modeling and Analysis of Multi-Phase Inverter Fed Induction Motor Drive with Different Phase Numbers. Transactions on Systems and Control, vol. 8, pp. 73-80, Jul. 2013.

[2] R. Dharmaprakash, J. Henry. High Performance Control Schemes of Induction Motor - A Review. International Journal of Advanced Research in Electrical, Electronics and Instrumentation Engineering, vol. 2, no. 9, pp. 4336-4347, 2013.

[3] A. Cifci, Y. Uyaroglu, S. Birbas. Direct Field Oriented Controller Applied to Observe Its Advantages over Scalar Control. Electronics and Eelectrical Engineering, vol. 119, no. 3, pp. 15-18, 2012.

[4] G. Renukadevi, K. Rajambal. FPGA Implementation of SVPWM Technique for Seven-Phase VSI. International Journal of Electronics and Electrical Engineering, vol. 1, no. 4, pp. 275-280, Dec. 2013.

[5] E. Levi, R. Bojoi, F. Profumo, H.A. Toliyat, S. Williamson. Multiphase Induction Motor Drives - A Technology Status Review. IET Electr. Power Appl., vol. 4, no. 1, pp. 489 -516, 2007.

[6] T. Lipinskis, A. Baskys, A. Rutkauskas. Six-Phase Voltage Forming Method Using the Largest Magnitude Space Vectors. Elektronika ir Elektrotechnika, vol. 19, no.10, pp. 99-102, 2013.

[7] G. Kohlrusz, D. Fodor. Comparison of Scalar and Vector Control Strategies of Induction Motors. Hungarian Journal of Industrial Chemistry, vol. 39, no. 2, pp. 265-270, 2011.

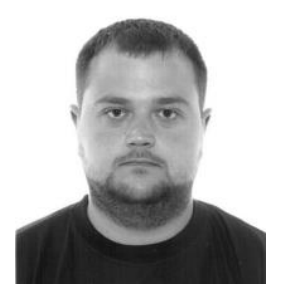

Aurelijus Pitrènas is a Ph.D. student at the department of Automation of Vilnius Gediminas Technical University (Lithuania) and works as an assistant in Vilnius Gediminas Technical University. He graduated B.Sc. M.Sc. degrees in electrical engineering, Vilnius Gediminas technical university in 2010 and 2012 respectively. Research interests: automated control systems, embedded systems, power electronics.

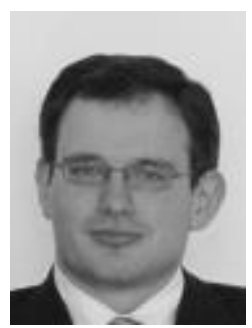

Andrius Petrovas recived the B.Sc.,M.Sc and $\mathrm{Ph} . \mathrm{D}$. degrees in electrical engineering from the Vilnius Gediminas technical university (Lithuania) in 2001, 2003 and 2007 respectively.

$\mathrm{He}$ works as Assoc. Prof. in Automation department of Vilnius Gediminas Technical University, since 2008. The area of scientific interests: automatic control theory, control and modeling of mechatronic systems. 